$2014 / 1$

\title{
The Application of the Level-crossing Theory of Multidimensional Random Processes to the Evaluation of Vehicle Operators' Performance
}

\author{
Aloizs Lesinskis, Institute of Aeronautics, Faculty of Transport and Mechanical Engineering, \\ Riga Technical University
}

\begin{abstract}
The application of the level-crossing theory of multidimensional random processes to the evaluation of the vehicle operators' performance allows not only evaluating the possible deviation from parameters, but also considering the dynamics of the parameter changes. The level-crossing intensity is acquired for normal distribution random process correlated by two dimensions, as well as for the multidimensional process when a maximum mutual correlation of parameters is considered. The criterion is defined that allows evaluating the possibility that none of the parameters will reach an unacceptable value.
\end{abstract}

Keywords - Criterion of movement safety, evaluation of operators' performance, level-crossing theory of multidimensional random processes.

\section{INTRODUCTION}

There are many reasons that determine the necessity for the evaluation of vehicle operators' (drivers, pilots etc.) performance - evaluation is used to estimate the initial practice progress during training, to issue respective licenses and certificates, and to assert qualification skills after a long hiatus etc. At present, the evaluation is subjective for the most part: by observing training or test run (drive, flight etc.) process and at best using objective control system data (if there is any).

When evaluating vehicle operators' performance, at least 3 aspects of their performance should be considered [1]:

1. manual regulation of uninterrupted movement parameters (steering, handling an airplane etc.);

2. discrete activities employing vehicle systems (turning on, turning off) - appropriateness, sequence and timeliness of such activities;

3. decision making about the change of trajectory or movement regime due to an actual situation (road signs, overtaking, interrupted taking off etc.).

The methods of evaluation are usually based on different norms - number of mistakes made (arranging them in the sequence of risk level), or movement parameter deviation from required values. The norms are mainly based on expert evaluation without objective, science-based value rationale; therefore, it is not possible to call an evaluation based on norms unbiased even though it is made automatically using objective control system data.

The most difficult one is the evaluation of the continuation of uninterrupted movement parameters, since we have to take into account not only momentary values of the parameters, but also the dynamics of their changes and the possibility that none of the parameters will reach an unacceptable catastrophic value. In fact, only when it is possible to estimate such probability or mathematically prove its direct correlation with the given criteria, only then we can claim that the evaluation is objective.

It is suggested using the level-crossing theory of multidimensional random processes to evaluate the continuation of uninterrupted movement parameters. It allows directly calculating the safety of vehicle movement possibility that during the movement none of the parameters will reach an unacceptable catastrophic value.

\section{LEVEL-CROSSING THEORY OF MULTIDIMENSIONAL RANDOM PROCESSES}

S. O. Rice was one of the first to develop the level-crossing theory of multidimensional random processes [2]. The systematic review and evolution of the theory and summary can be found in [3] - [8].

The aim of the theory is to find statistic parameters of random process $\boldsymbol{x}(\boldsymbol{t})$ that are connected with the crossing of specific level $c$ (see Fig. 1): the moment of first level-crossing $\left(\boldsymbol{\tau}_{\boldsymbol{0}}\right)$, time of level-crossing $(\boldsymbol{\tau})$, local $(\boldsymbol{H})$ and absolute $\left(\boldsymbol{H}_{\boldsymbol{m}}\right)$ maximum, duration of location under level $\boldsymbol{c}-(\boldsymbol{\Theta})$ etc.

One of the most used parameters is level-crossing intensity $\lambda_{c}(t)$ - an average number of level-crossings during the evaluated interval and its characteristics (distribution etc.).

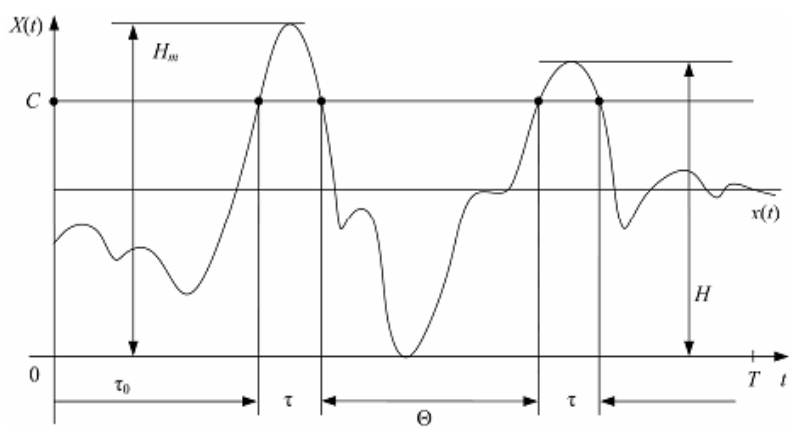

Fig. 1. Characteristic parameters of level-crossing of random processes.

Crossing with positive process gradient $\boldsymbol{d x}(\boldsymbol{t}) / \boldsymbol{d t}>\mathbf{0}$ (see Fig. 2) over level $c$ that is above the mathematical expectation of process $\boldsymbol{\mu}(\boldsymbol{t})$ is called positive level-crossing. The negative level-crossing is defined in the analogous fashion. 


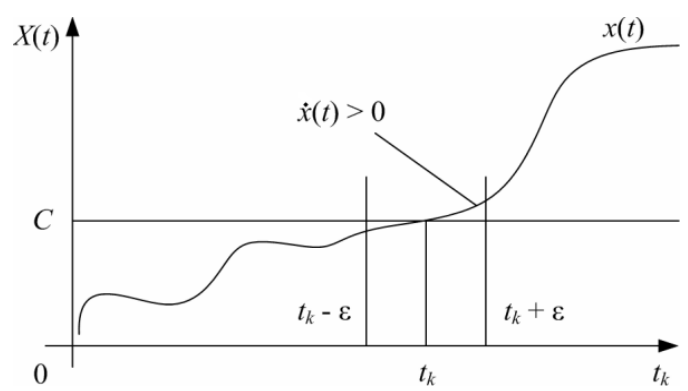

Fig. 2. Diagram of the positive level-crossing of random processes.

The number of level-crossings in time interval $0 \ldots T$ is defined by coherence:

$$
\begin{aligned}
& N_{c}(T)=\int_{0}^{T} \delta(x(t)-c) \cdot 1\left(\frac{d x(t)}{d t}\right) d x= \\
& =\int_{0}^{T} \dot{x}(t) \delta(x(t)-c) \cdot 1(\dot{x}(t)) \cdot d t
\end{aligned}
$$

where $\delta(\ldots)$ - the delta function, but $1(\ldots)$ - the Heaviside function.

Indeed, on points $t_{k}(\mathrm{k}=1,2, \ldots)$ an integral function is infinite (since $\delta(0)=\infty$ ) and on each of these points the integral increases in leaps by 1 according to the Heaviside function.

Mathematical expectation of the level-crossing number:

$$
\begin{aligned}
M\left[N_{c}(T)\right]= & \int_{0}^{\infty} \int_{0}^{\infty} N_{c}(T, x(t), \dot{x}(t)) \times \\
& \times f(x(t), \dot{x}(t)) d x d \dot{x}
\end{aligned}
$$

where $f(x(t), \dot{x}(t))-\boldsymbol{x}(t)$ and function of its derivative inclusive distribution.

Inserting (1) into (2) and applying the established coherence for the integration of some function multiplication with delta function (11), by integrating after $\boldsymbol{x}$ we obtain:

$$
M\left[N_{c}(T)\right]=\int_{0}^{T} \int_{0}^{\infty} \dot{x}(t) f(c, \dot{x}(t)) d \dot{x} d t
$$

If the process is stationary in the narrow sense, normal in distribution and differentiable, then its distribution function is not conditioned by time, the process and its derivatives have no mutual correlation, and the mathematical expectation of derivative $=0$ :

$$
f(x(t), \dot{x}(t))=\frac{1}{2 \pi \sigma_{x} \sigma_{\dot{x}}} e^{-\frac{1}{2}\left(\frac{(x-\mu)^{2}}{\sigma_{x}^{2}}+\frac{(\dot{x})^{2}}{\sigma_{\dot{x}}^{2}}\right)}
$$

where $\boldsymbol{\mu}-x(t)$ mathematical expectation, but $\boldsymbol{\sigma}_{\boldsymbol{x}}$ and $\boldsymbol{\sigma}_{\dot{\boldsymbol{x}}}$ are average square deviations of the process and its derivative, respectively.

Inserting (4) into (3) and then integrating we obtain:

$$
M\left[N_{c}(T)\right]=\frac{T \cdot \sigma_{\dot{x}}}{2 \pi \sigma_{x}} e^{-\frac{1(c-\mu)^{2}}{2} \sigma_{x}^{2}}
$$

Level-crossing intensity or the average number of levelcrossings in the time interval is obtained, dividing (5) by $\mathrm{T}$ :

$$
\lambda_{c}(T)=\frac{\sigma_{\dot{x}}}{2 \pi \sigma_{x}} e^{-\frac{1(c-\mu)^{2}}{2} \sigma_{x}^{2}}
$$

If $c-\mu>(2 . .3) \sigma$, then level-crossings are discrete, independent events and are governed by Poisson distribution. Probability that in interval $0 \ldots T$ there will be $\boldsymbol{m}$ levelcrossings is as follows [3, 9]:

$$
P=P\left(N_{c}(T)=m\right)=\frac{\left[\lambda_{c}(T) \cdot T\right]^{m}}{m !} e^{-\left[\lambda_{c}(T)\right] \cdot T}
$$

Probability that in interval $0 \ldots T$ there will not be any levelcrossing is as follows:

$$
P=e^{-\lambda_{c} T}
$$

By analogy the probability of the negative (downward moving) level-crossing can be calculated. However, these equations are not sufficient for the estimation of the level-crossing intensity of the vehicle movement parameters and movement safety. The vehicle movement is usually characterised not just by one, but several movement parameters that are regulated by the operator, and these parameters in many instances are not mutually independent but are more or less in correlation.

Let us formulate the task for the calculation of the number of level-crossings of the multidimensional correlated random processes.

\section{ASSUMPTIONS - HYPOTHESES}

1. Performance of the operator in regulating uninterrupted parameters is governed by normal distribution of multidimensional random process. This hypothesis can be based on the central limit theorem [10, 11] of probability theory that basically states that if the process is influenced by a large number of variables (their sum) and none of them is dominant, then the process will be approximately normally distributed regardless of the actual distribution laws of its components. The 
parameters of the vehicle movement, indeed, are influenced by a large number of variables: for example, atmospheric stimulation, conditions of the road, psychophysical state of an operator, flow of information and its processing etc. Although it is not possible to assure that in all instances the process is normally distributed, exactly this can be regarded as the most common occurrence and, thus, the first to be studied.

2. Movement itinerary can be divided into stationary phases. Basically the trajectory of any vehicle consists of stationary phases during which the movement from point A to point $B$ is carried out under invariable spectre of external stimulation and limits. There is no reason why the movement in these phases should not be considered stationary.

3. During non-stationary stages a particular type of nonstationarity can be identified that depends on the tasks to be accomplished during the phase. It is common for all trained operators. The transition processes from one phase to another, of course, are non-stationary. However, these phases in many instances can be regarded as stationary by centring them on one dominant nonstationarity. For the most part, the proportion of nonstationary stages during the interval is small in comparison with the stationary stages, but their significance is remarkable and the probability that during those particular stages a critical value of parameters will be exceeded is huge. Separate study is needed to identify which mathematical model and theory will be the most appropriate here.

4. Performance of the operator in regulating uninterrupted parameters is a differentiable random process. The movement of the vehicle can be described by a corresponding number of differential equations. Therefore, the process is differentiable. The movements of the operator while operating the vehicle can also be regarded as a differentiable process.

5. Performance of the operator in regulating uninterrupted parameters is an ergodic random process. If the values of the controllable parameters are defined and limits are symmetrical in relation to these parameters, then there is no reason to assume that the operator will deliberately try to disregard the necessary values and the process will not be ergodic. Mathematical expectation, for example, in each realization will be individual. The divergence from the recommended mathematical expectation can be related to the manner of driving (for example, inclination to exceed the allowed speed limit by a specific value, keeping closer to the roadside), yet it changes comparatively slowly, except perhaps initial training. These are a few arguments in favour of ergodic process hypothesis.

\section{The TASK OF Estimating THE NuMBer OF LEVEL- CROSSINGS OF THE MULTIDIMENSIONAL CORRELATED RANDOM PROCESS}

$\boldsymbol{n}$-dimension random process vector $[12,13] \boldsymbol{X}$ is given with stationary functions $x_{1}(t), x_{2}(t), \ldots, x_{n}(t)$ that correspond to normal distribution with mathematical expectations $\boldsymbol{\mu}_{\boldsymbol{l}}$, $\boldsymbol{\mu}_{2}, \ldots, \boldsymbol{\mu}_{\boldsymbol{n}}$ and correlation matrix $\boldsymbol{K}=\left\|\boldsymbol{k}_{i j}\right\|$, where $\boldsymbol{i}=\mathbf{1}, 2, \ldots, \boldsymbol{n}$; $j=1,2, \ldots, n ; k_{i j}=\sigma_{i} \sigma_{j} r_{i j} ; \sigma_{i}, \sigma_{j}-$ the average square deviation of processes $\boldsymbol{x}_{i}(\boldsymbol{t})$ and $\boldsymbol{x}_{i}(\boldsymbol{t})$, but $\boldsymbol{r}_{i j}$ - the mutual correlation coefficient of processes $\boldsymbol{x}_{i}(t)$ and $\boldsymbol{x}_{i}(t)$.

$\boldsymbol{n}$ dimension limit area $\boldsymbol{C}$ is also defined and it is given to each dimension of process $\boldsymbol{x}_{\boldsymbol{i}}(\boldsymbol{t})$ with lower $\boldsymbol{c}_{\boldsymbol{i L}}$ and upper $\boldsymbol{c}_{\boldsymbol{i} \boldsymbol{H}}$ levels, and the crossing of these levels will be considered level-crossing.

Probability $\boldsymbol{P}$ should be calculated for an instance when during interval $\boldsymbol{t}_{\boldsymbol{o}} \ldots \boldsymbol{t}_{\boldsymbol{o}}+\boldsymbol{T}$ process $\boldsymbol{X}$ will not cross limit area $\boldsymbol{C}$.

Likewise an one-dimensional process, level-crossing intensity $\lambda$ must be found - the number of level-crossings during the interval that in case of $c-\mu>(2 . .3) \sigma_{x}$ usually corresponds to Poisson distribution.

For the realization of the specific process, level-crossing number $\boldsymbol{v}$ of vector $\boldsymbol{X}$ outside the area $\boldsymbol{C}$ can be calculated as the sum of these events: level-crossing for all processes $\boldsymbol{x}_{i}(\boldsymbol{t})$ crossing its upper limit $\boldsymbol{c}_{i \boldsymbol{H}}$ with positive derivation of this function $\boldsymbol{n}_{t}(t)$ plus the crossing of lower limit $c_{i L}$ with negative derivation $\boldsymbol{x}_{i}(t)$ on condition that other functions $\boldsymbol{n}-\boldsymbol{1}$ of the vector are at the same time located within area $\boldsymbol{C}$.

$$
\begin{aligned}
& v=\sum_{i=1}^{n}\left(\int_{t_{0}}^{t_{0}+T}\left(\dot{x}_{i}^{+}(t) \delta\left(x_{i}(t)-c_{i H}\right) \mid X_{n-1} \in C\right) d t+\right. \\
& \left.+\int_{t_{0}}^{t_{0}+T}\left(-\dot{x}_{i}^{-}(t) \delta\left(x_{i}(t)-c_{i L}\right) \mid X_{n-1} \in C\right) d t\right)
\end{aligned}
$$

Since the average number of level-crossings during the interval is a random number, the level-crossing intensity can be found as mathematical expectation $\boldsymbol{T}=1$ of level-crossing number $\boldsymbol{v}$ :

$$
\begin{aligned}
& \lambda\left(t_{0}\right)=\sum_{i=1}^{n}\left(\int _ { t _ { 0 } } ^ { t _ { 0 } + 1 } \int _ { c _ { 1 L } } ^ { c _ { 1 H } } \int _ { c _ { 2 L } } ^ { c _ { 2 H } } \ldots \int _ { c _ { i L } } ^ { c _ { i H } } \ldots \int _ { c _ { n L } } ^ { c _ { n H } } \left(\int_{0}^{+\infty} \dot{x}_{i}(t) \delta\left(x_{i}(t)-c_{i H}\right)+\right.\right. \\
& \left.+\int_{-\infty}^{0}\left(-\dot{x}_{i}(t) \delta\left(x_{i}(t)-c_{i H}\right)\right)\right) f_{n+1}\left(\dot{x}_{i}(t), x_{1}(t), x_{2}(t), \ldots\right. \\
& \left.\left.\ldots, x_{i}(t), \ldots, x_{n}(t)\right) d t d x_{1} d x_{2} \ldots d x_{i} \ldots d x_{n} d \dot{x}_{i}\right)
\end{aligned}
$$

$$
\begin{aligned}
& \text { where } f_{n+1}\left(\dot{x}_{i}(t), x_{1}(t), x_{2}(t), \ldots, x_{i}(t), \ldots, x_{n}(t)\right) \text { is a } \\
& \text { common distribution density function } \\
& \dot{x}_{i}(t), x_{1}(t), x_{2}(t), \ldots, x_{i}(t), \ldots, x_{n}(t) \text { functions. }
\end{aligned}
$$


$\boldsymbol{x}_{i}(\boldsymbol{t})$ integration can be done by using coherence for the intergration with $\boldsymbol{\delta}$ function:

$$
\int_{z_{0}-\varepsilon}^{z_{0}+\varepsilon} f(z) \delta\left(z-z_{0}\right) d z=f\left(z_{0}\right)
$$

By integrating after $\boldsymbol{x}_{\boldsymbol{i}}$, we obtain:

$$
\begin{aligned}
& \lambda\left(t_{0}\right)=\sum_{i=1}^{n}\left(\int _ { t _ { 0 } } ^ { t _ { 0 } + 1 } \int _ { c _ { 1 L } } ^ { c _ { 1 H } } \int _ { c _ { 2 L } } ^ { c _ { 2 H } } \ldots \int _ { c _ { i - 1 L } } ^ { c _ { i - 1 H } } \int _ { c _ { i + 1 L } } ^ { c _ { i + 1 H } } \ldots \int _ { c _ { n L } } ^ { c _ { n H } } \left(\int_{0}^{+\infty} \dot{x}_{i}(t) \times\right.\right. \\
& \times f_{n+1}\left(\dot{x}_{i}(t), x_{1}(t), x_{2}(t), \ldots, x_{i-1}(t), c_{i H}, x_{i+1}(t), \ldots, x_{n}(t)\right)+ \\
& +\int_{0}^{-\infty} \dot{x}_{i}(t) f_{n+1}\left(\dot{x}_{i}(t), x_{1}(t), x_{2}(t), \ldots, x_{i-1}(t), c_{i L}, x_{i+1}(t), \ldots\right. \\
& \left.\left.\ldots, x_{n}(t)\right) d t d x_{1} d x_{2} \ldots d x_{i-1} d x_{i+1} \ldots d x_{n} d \dot{x}_{i}\right)
\end{aligned}
$$

It should be pointed out that up till now coherences are valid not only for the normal distribution and not only for the stationary process.

If we assume that the process is stationary and $\dot{x}_{i}$ has no correlation with $\boldsymbol{X}$, then previous coherence can be written as follows:

$$
\begin{aligned}
& \lambda=\sum_{i=1}^{n}\left(\int _ { c _ { 1 L } } ^ { c _ { 1 H } } \int _ { c _ { 2 L } } ^ { c _ { 2 H } } \ldots \int _ { c _ { i - 1 L } } ^ { c _ { i - 1 H } } \int _ { c _ { i + 1 L } } ^ { c _ { i + 1 H } } \ldots \int _ { c _ { n L } } ^ { c _ { n H } } \left(\int_{0}^{+\infty} \dot{x}_{i} f\left(\dot{x}_{i}\right) .\right.\right. \\
& f_{n}\left(x_{1}, x_{2}, \ldots, x_{i-1}, c_{i H}, x_{i+1}, \ldots, x_{n}\right)+ \\
& +\int_{0}^{-\infty} \dot{x}_{i} f\left(\dot{x}_{i}\right) f_{n}\left(x_{1}, x_{2}, \ldots, x_{i-1}, c_{i L}, x_{i+1}, \ldots\right. \\
& \left.\left.\left.\ldots, x_{n}\right)\right) d x_{1} d x_{2} \ldots d x_{i-1} d x_{i+1} \ldots d x_{n} d \dot{x}_{i}\right)
\end{aligned}
$$

where $f\left(\dot{x}_{i}\right)-\dot{x}_{i}$ distribution density function.

The author managed to integrate (13) into the normal, stationary process in 2 cases only if:

1. the process has only 2 dimensions;

2. the dimensions of the process are mutually independent (non-correlated).

\section{The PATtern OF the RANDOM PROCESS LEVEL-CROSSING CORRELATED BY TWO DIMENSIONS}

Stationary differentiable normal distribution random processes $\boldsymbol{x}_{1}(\boldsymbol{t})$ and $\boldsymbol{x}_{2}(\boldsymbol{t})$ are given with the mathematical expectation, average square deviation and derivation's average square deviation respectively $\mu_{1}, \sigma_{1}, \quad$ and $\mu_{2}, \sigma_{2}$, , as well as with mutual correlation coefficient $\boldsymbol{r}$ and distribution density function:

$$
\begin{gathered}
f\left(x_{1}, x_{2}\right)=\frac{1}{2 \pi \sigma_{1} \sigma_{2} \sqrt{1-r^{2}}} \exp \left[-\frac{1}{2\left(1-r^{2}\right)}\left(\frac{\left(x_{1}-\mu_{1}\right)^{2}}{\sigma_{1}^{2}}-\right.\right. \\
\left.\left.-\frac{2 r\left(x_{1}-\mu_{1}\right)\left(x_{2}-\mu_{2}\right)}{\sigma_{1} \sigma_{2}}+\frac{\left(x_{2}-\mu_{2}\right)^{2}}{\sigma_{2}^{2}}\right)\right]
\end{gathered}
$$

Likewise, lower $\left(\boldsymbol{c}_{1 L}, \boldsymbol{c}_{2 L}\right)$ and upper $\left(\boldsymbol{c}_{1 H}, \boldsymbol{c}_{2 H}\right)$ levels of each process are given. Crossing outside those levels will be considered level-crossing (see Fig. 3).

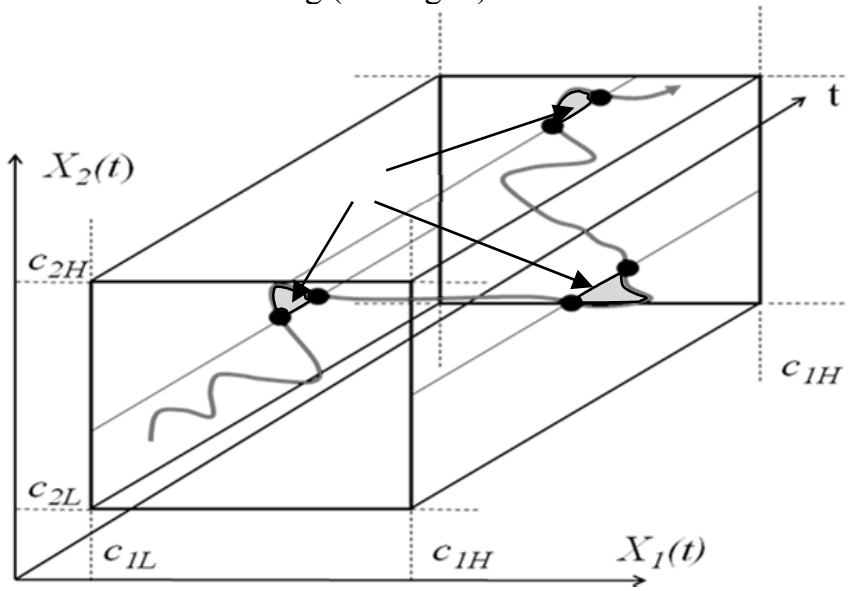

Fig. 3. Level-crossing pattern of two-dimensional random process from a fixed rectangular corridor.

By inserting (14) into (13) after integrating we obtain the level-crossing number intensity for the correlated twodimensional normal stationary process from rectangular corridor $c_{1 L} \ldots c_{1 H}, c_{2 L} \ldots c_{2 H}$ :

$$
\begin{aligned}
& \lambda=\frac{\sigma_{\dot{x}_{1}}}{2 \pi \sigma_{1}}\left(e^{-\frac{1}{2}\left(\frac{c_{1 H}-\mu_{1}}{\sigma_{1}}\right)^{2}} \times\left(\Phi ^ { * } \left(\frac { 1 } { \sqrt { 1 - r ^ { 2 } } } \left(\frac{c_{2 H}-\mu_{2}}{\sigma_{2}}-\right.\right.\right.\right. \\
& \left.\left.\left.-r \frac{c_{1 H}-\mu_{1}}{\sigma_{1}}\right)\right)-\Phi^{*}\left(\frac{1}{\sqrt{1-r^{2}}}\left(\frac{c_{2 L}-\mu_{2}}{\sigma_{2}}-r \frac{c_{1 H}-\mu_{1}}{\sigma_{1}}\right)\right)\right)+ \\
& +e^{-\frac{1}{2}\left(\frac{c_{1 L}-\mu_{1}}{\sigma_{1}}\right)^{2}} \times\left(\Phi^{*}\left(\frac{1}{\sqrt{1-r^{2}}}\left(\frac{c_{2 H}-\mu_{2}}{\sigma_{2}}-r \frac{c_{1 L}-\mu_{1}}{\sigma_{1}}\right)\right)-\right. \\
& \left.\left.-\Phi^{*}\left(\frac{1}{\sqrt{1-r^{2}}}\left(\frac{c_{2 L}-\mu_{2}}{\sigma_{2}}-r \frac{c_{1 L}-\mu_{1}}{\sigma_{1}}\right)\right)\right)\right)+ \\
& -\sigma_{\dot{x}_{2}}\left(e^{-\frac{1}{2}\left(\frac{c_{2 H}-\mu_{2}}{\sigma_{2}}\right)^{2}} \times\left(\Phi ^ { * } \left(\frac { 1 } { \sqrt { 1 - r ^ { 2 } } } \left(\frac{c_{1 H}-\mu_{1}}{\sigma_{1}}-\right.\right.\right.\right. \\
& \left.\left.\left.-r \frac{c_{2 H}-\mu_{2}}{\sigma_{2}}\right)\right)-\Phi^{*}\left(\frac{1}{\sqrt{1-r^{2}}}\left(\frac{c_{1 L}-\mu_{1}}{\sigma_{1}}-r \frac{c_{2 H}-\mu_{2}}{\sigma_{2}}\right)\right)\right)+ \\
& +e^{-\frac{1}{2}\left(\frac{c_{2 L}-\mu_{2}}{\sigma_{2}}\right)^{2}} \times\left(\Phi ^ { * } \left(\frac { 1 } { \sqrt { 1 - r ^ { 2 } } } \left(\frac{c_{1 H}-\mu_{1}}{\sigma_{1}}-\right.\right.\right. \\
& \left.\left.\left.\left.-r \frac{c_{2 L}-\mu_{2}}{\sigma_{2}}\right)\right)-\Phi^{*}\left(\frac{1}{\sqrt{1-r^{2}}}\left(\frac{c_{1 L}-\mu_{1}}{\sigma_{1}}-r \frac{c_{2 L}-\mu_{2}}{\sigma_{2}}\right)\right)\right)\right)
\end{aligned}
$$


where $\Phi^{*}(x)=\frac{1}{2 \pi} \int_{-\infty}^{x} e^{-t^{2} / 2} d t-$ the probability integral.

\section{LEVEL-CROSSING OF THE NON-CORRELATED MULTIDIMENSIONAL NORMAL STATIONARY PROCESS}

For the non-correlated multidimensional normal stationary process, a distribution density function is a multiplication of distribution density functions of separate dimension:

$$
f_{n}\left(x_{1}, x_{2}, \ldots, x_{n}\right)=\prod_{i=1}^{n} \frac{1}{2 \pi \sigma_{i}} e^{-\frac{1}{2}\left(\frac{\left(x_{i}-\mu_{i}\right)^{2}}{\sigma_{i}^{2}}\right)}
$$

By using (16) in (13) after integration we obtain the levelcrossing number intensity for the non-correlated multidimensional normal stationary process from limited area C:

$$
\begin{aligned}
\lambda= & \sum_{i=1}^{n}\left(\frac{\sigma_{\dot{x}_{i}}}{2 \pi \sigma_{i}}\left(e^{-\frac{1}{2}\left(\frac{c_{i H}-\mu_{i}}{\sigma_{i}}\right)^{2}}+e^{-\frac{1}{2}\left(\frac{c_{i L}-\mu_{i}}{\sigma_{i}}\right)^{2}}\right) \times\right. \\
& \left.\times \prod_{\substack{k=1 \\
k \neq i}}^{n}\left(\Phi^{*}\left(\frac{c_{k H}-\mu_{k}}{\sigma_{k}}\right)-\Phi^{*}\left(\frac{c_{k L}-\mu_{k}}{\sigma_{k}}\right)\right)\right)
\end{aligned}
$$

\section{The PATtern OF APproximate CALCUlation OF THE LEVEL-CROSSING INTENSITY IN THE CORRELATED MulTidimensional NoRmal STATIONERY PROCESS}

The number of the level-crossings of the correlated multidimensional normal stationary process can be calculated approximately on condition that for each variable $(\boldsymbol{i})$ of the process we regard only its maximal correlation with some other variable $(\boldsymbol{j})-\boldsymbol{r}_{i j}$ and other coefficients of the correlation for the duration of the calculation are assumed as zero.

In this case functions $f_{n}\left(x_{1}, x_{2}, \ldots, x_{i-1}, c_{i H}, x_{i+1}, \ldots, x_{n}\right)$ and $f_{n}\left(x_{1}, x_{2}, \ldots, x_{i-1}, c_{i L}, x_{i+1}, \ldots, x_{n}\right)$ can be respectively taken as: $f_{i j}\left(c_{i H}, x_{j}\right) \prod_{\substack{k=1 \\(k \neq i) \\ k \neq j}}^{n} f\left(x_{k}\right)$ and $f_{i j}\left(c_{i L}, x_{j}\right) \prod_{\substack{k=1 \\ k \neq i) \\ k \neq j}}^{n} f\left(x_{k}\right)$

By inserting (18) into (13), after integration we obtain:

$$
\begin{aligned}
& \lambda=\sum_{i=1}^{n}\left(\frac { \sigma _ { \dot { x } _ { i } } } { 2 \pi \sigma _ { i } } \left(e^{-\frac{1}{2}\left(\frac{c_{i H}-\mu_{i}}{\sigma_{i}}\right)^{2}} \times\left(\Phi ^ { * } \left(\frac { 1 } { \sqrt { 1 - r _ { i j } ^ { 2 } } } \left(\frac{c_{j H}-\mu_{j}}{\sigma j}-\right.\right.\right.\right.\right. \\
& \left.\left.\left.-r_{i j} \frac{c_{i H}-\mu_{i}}{\sigma_{i}}\right)\right)-\Phi^{*}\left(\frac{1}{\sqrt{1-r_{i j}^{2}}}\left(\frac{c_{j L}-\mu_{j}}{\sigma_{j}}-r_{i j} \frac{c_{i H}-\mu_{i}}{\sigma_{i}}\right)\right)\right)+ \\
& +e^{-\frac{1}{2}\left(\frac{c_{i L}-\mu_{i}}{\sigma_{i}}\right)^{2}} \times\left(\Phi^{*}\left(\frac{1}{\sqrt{1-r_{i j}^{2}}}\left(\frac{c_{j H}-\mu_{j}}{\sigma_{j}}-r_{i j} \frac{c_{i L}-\mu_{i}}{\sigma_{i}}\right)\right)-\right. \\
& \left.\left.-\Phi^{*}\left(\frac{1}{\sqrt{1-r_{i j}^{2}}}\left(\frac{c_{j L}-\mu_{j}}{\sigma j}-r_{i j} \frac{c_{i L}-\mu_{i}}{\sigma_{i}}\right)\right)\right)\right) \times \\
& \left.\times \prod_{\substack{k=1 \\
(k \neq i) \\
(k \neq j)}}^{n}\left(\Phi^{*}\left(\frac{c_{k H}-\mu_{k}}{\sigma_{k}}\right)-\Phi^{*}\left(\frac{c_{k L}-\mu_{k}}{\sigma_{k}}\right)\right)\right)
\end{aligned}
$$

Probability that there will not be any level-crossing during interval $0 \ldots T$ in accordance with (7) and (8) is as follows:

$$
P=e^{-\lambda T}
$$

\section{THE MODELLING OF THE EVALUATION CRITERION}

According to (20), probability $\boldsymbol{P}$ decreases while $\boldsymbol{T}$ increases. It is true since with $\lambda$ one of the critical limits will be reached sooner or later and an absolutely safe movement with the random process features does not exist.

However, (20) cannot be used in this way as the evaluation criterion for judging operator's performance because:

- the evaluation criterion must be comparable in order to follow up the operator's qualification dynamics and carry out its comparative analysis with other operators;

- since $\lambda$ is conditioned not only by operator's performance but also by external stimulation during a particular run, the evaluation must be carried out in equivalent conditions and circumstances;

- the criterion should be maximally sensitive to the qualification of the operator and greater emphasis should be placed on those movement phases during which operators make most mistakes or where the consequences of these mistakes are the gravest.

The equivalent conditions and external stimulations can be ensured most easily by using vehicle simulators. It is also the easiest way to organize the registration of the run with the necessary precision, though it is not impossible on the real vehicles.

Aviation [14] is the most suitable vehicle sphere with trueto-life simulators, as well as nowadays airplanes are equipped with flight registration tools and easily accessible information on atmosphere conditions.

Let us examine how it is possible to use (20) in the evaluation of the airplane piloting taking into account the above-mentioned requirements. 
For the evaluation of the flight phase importance we will calculate importance coefficient $\boldsymbol{k}_{\boldsymbol{p} \boldsymbol{h}}$ of the phase:

$$
k_{p h}=\frac{n_{p h}}{n_{\text {sum }}}
$$

where $\boldsymbol{n}_{\boldsymbol{p} \boldsymbol{h}}-$ an average number of flight accidents during a respective phase, but $\boldsymbol{n}_{\text {sum }}$ - the sum of accidents that in the corresponding airplane class are related to piloting.

For each phase we will assign equal realization time in accordance with its importance coefficient so that the total interval will be 1 hour $=3600$ seconds. As a result, we can calculate the safety of piloting during one respective flight hour:

$$
P_{h}=e^{-\sum_{(p h)} \lambda_{p h} k_{p h} 3600}
$$

where $\lambda_{p h}$ - the level-crossing intensity of the phase calculated in accordance with (19).

The influence of the particular flight conditions can be excluded if (22) is rationed according to the standard flight in analogous circumstances:

$$
P_{c r}=\frac{P_{h}}{P_{h s t}}
$$

where $\boldsymbol{P}_{\boldsymbol{h} \text { st }}$ - theoretical flight piloting safety calculated in accordance with (22) for the flight crews with the standard piloting technique on which other activities in particular circumstances should be based.

$\boldsymbol{P}_{h s t}$ can be obtained by statistically processing the flights of best flight crews.

$\boldsymbol{P}_{\boldsymbol{c} r}$ signification can be defined as follows: it is a probability that none of the flight parameters will cross the acceptable limit during the respective flight hour; if we assume that a standard flight crew journey in similar circumstances is absolutely safe.

In some cases $\boldsymbol{P}_{\boldsymbol{c} r}$ can assume value $>1$. If this occurs too often the statistics of standard crews should be re-evaluated and $\boldsymbol{P}_{h s t}$ increased.

\section{CONCLUSIONS}

It is preferable to calculate the values of the evaluation criterion during the flight and it is recommended acquiring the evaluation immediately after the end of the phase or at least after the flight. It can be accomplished by using recurrent calculation equations for the calculation of evaluations of the necessary statistic moments.

For the identification of the beginning and end stages of the phase, particular algorithms often should be employed with the possibility to return back to the beginning of the identification since it is probable that manoeuvre considered to be a start of the next phase in fact does not start the next phase but instead continues the movement of the previous phase.

If there is a necessity to evaluate each individual flight, most of the time individual realizations of the stationary phases are too short to acquire statistically valid distribution parameters from one realization. On the other hand, each individual flight should be evaluated, especially in qualification tests. In order to increase the precision of the statistic parameter identification, the methods of the small sample processing that is based on maximum usage of prior information should be employed [1]. It remains to be separately researched as to which information in particular circumstances can be considered a priori known given that it can decrease an efficiency of the evaluation by equalizing all operators via one particular parameter or by hiding the dynamics of the skill development [15] if the value of the parameter is acquired by processing many successive flights of one operator.

Even though the criterion of the movement safety evaluation can be regarded as unbiased, it is nevertheless difficult to explain and comprehend for the vehicles operators. However the explanation should be given in order to clarify which particular mistakes were made and what specific actions should be carried out in order to prevent them. Therefore, the combination of the criterion with the announcements on a dangerous approach to the limits etc. is necessary.

Coherences (15), (17) and (19) can be used also in other spheres of engineering sciences in cases when it is necessary to calculate level-crossing intensity of the normal distribution stationary differentiable correlated multidimensional process in a linear limited area.

\section{ACKNOWLEDGEMENTS}

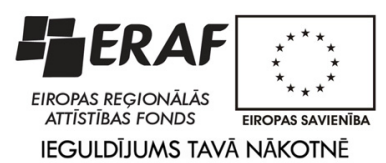

The research has been supported by the European Regional Development Fund within the framework of project "Development of an Unmanned Aircraft System and Creation of the Industrial Prototypes of Unmanned Aerial Vehicles for Performing the Tasks of Latvian National Economy" No.2010/0256/2DP/2.1.1.1.0/10/APIA/VIAA/070

\section{REFERENCES}

[1] A. Lešinskis, A. Urbahs and P. Vulans, Statistical Methods of Performance Evaluation of the Vehicle Operators and the Related Problem of Small Sample: 17th International Conference “Mechanika'2012”, pp. 169-175, Apr.12-13, 2012, Kaunas, Lithuania.

[2] S. O. Rice, "Mathematical analysis of random noise," Bell System Technical Journal, vol. 23, pp. 282-332, 1944. http://dx.doi.org/10.1002/j.1538-7305.1944.tb00874.x

[3] V. I. Tihonov, Vybrosy slucajnyh processov. Moskva: Nauka, 1970.

[4] M. Kratz, "Level crossings and other level functionals of stationary Gaussian processes," Probability Surveys, vol.3, pp. 230-288, 2006. http://dx.doi.org/10.1214/154957806000000087

[5] I. F. Blake and W. C. Lindsey, "Level-crossing problems for random processes," IEEE Transactions on Information Theory, vol. 19, pp. 295$315,1973$.

http://dx.doi.org/10.1109/TIT.1973.1055016 
$2014 / 1$

[6] A. Y. Fomin, Teoria vybrosov slucajnyh processov. Moskva: Svaz, 1980.

[7] V.I. Tihonov and V. I. Himenko, Vybrosy traektorij slucajnyh processov. Moskva: Nauka, 1987.

[8] T. Munakata and D. Wolf, A novel approach to the level crossing problem of random processes: IEEE International Symposium on Information Theory, pp. 149-150, June 21-25, 1982, Les Arcs, France.

[9] V. I Piterbarg, "The central limit theorem for the number of level crossings of a stationary Gaussian process," Theory of Probability \& Its application, vol.23, pp. 178-182, 1978.

[10] J. S. Bendat and A. G. Piersol, Random Data Analysis and Measurement Procedures. New York: Wiley, 2010. http://dx.doi.org/10.1002/9781118032428

[11] L. Giraitis and D. Surgailis, "CLT and other Limit Theorems for Functionals of Gaussian Processes", Probability Theory and Related Fields, vol. 70, no. 2, pp. 191-212, 1985.

[12] V. Chuberkis, "Crossings of fixed levels by two stationary random processes", Automatic Remote Control, vol 32, pp. 1581-1587, 1971.

[13] R. J. Adler, "Excursions above a Fixed Level by n-dimensional Random Fields," Journal of Applied Probability, vol 13, pp. 276-289, 1976. http://dx.doi.org/10.2307/3212831

[14] S. L. Semakov, Vybrosy slucajnyh processov: prilozenia $v$ aviacii. Moskva, Nauka, 2005.

[15] A. Lešinskis, Assessment of Aircraft Crew Skills Development Dynamics by Means of a Separate Exercise Results: Riga Technical University 53rd International Scientific Conference, pp. 620, Oct. 11-12, 2012, Riga, Latvia.

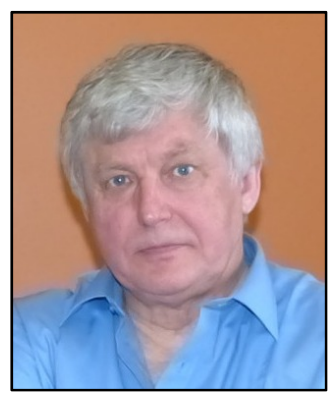

Aloizs Lesinskis graduated from the Faculty of Mechanical Engineering of Riga Civil Aviation Institute in 1975 and was awarded the degree of Master of Engineering Science by Riga Aviation University in 1996. Work experience: since 2012 - Assistant Professor at the Institute of Aeronautics, Riga Technical University; from 2001 to 2012 - Assistant Professor at Riga International School of Economics and Business Administration (RISEBA); from 1999 to 2001 - Lecturer at Riga Technical University; from 1992 to 1999 - Lecturer at Riga Aviation University; from 1975 to $1992-$ Engineer and Senior Researcher at Riga Civil Aviation Institute.

His fields of research: computer-aided training system, aerodynamics, flight dynamics, aviation ergonomics, supervisory control and data acquisition (SCADA), telemetry systems, information technology applications, analysis and modelling of complex systems, decision support systems.

Institute of Aeronautics, Faculty of Transport and Mechanical Engineering,

Riga Technical University, Lomonosova 1A, k-1, Riga, LV-1019, Latvia.

Phone: +371 67089990

E-mail: Aloizs.Lesinskis@rtu.lv 